\title{
How to measure respiratory mechanics during controlled mechanical ventilation
}

\author{
Marco Giani ${ }^{1,2}$, Alfio Bronco ${ }^{1}$, Giacomo Bellani ${ }^{1,2}$ \\ ${ }^{1}$ Dipartimento di Emergenza-Urgenza, ASST Monza, Monza - Italy \\ ${ }^{2}$ Dipartimento di Medicina e Chirurgia, Università degli Studi di Milano-Bicocca, Monza - Italy
}

\begin{abstract}
Bedside measurement of respiratory mechanics allows to closely monitor the lung function in critically ill patients. The two fundamental parameters describing the respiratory system mechanics are resistance and compliance. Resistance of the respiratory system describes the opposition to gas flow during inspiration. During volumecontrolled ventilation, resistance can be calculated as the ratio between the peak to plateau pressure drop and the resulting flow rate. Compliance describes the elastic property of the respiratory system, comprising the lung and the chest wall. It is the ratio between a change in volume (i.e. tidal volume) and the corresponding change in pressure, calculated as the difference between plateau pressure and total positive end-expiratory pressure, measured by end-inspiratory and end-expiratory manual occlusion, respectively. In this review, we describe how to measure respiratory mechanics at the bedside, starting from the physiological background of the equation of motion of the respiratory system.
\end{abstract}

Keywords: Compliance, Mechanical ventilation, Resistance, Respiratory mechanics

\section{Introduction}

Measurement of respiratory mechanics provides information about the alteration of lung and respiratory system homeostasis through analyses of their mechanical properties during mechanical ventilation $(1,2)$. The bedside assessment of mechanics in ventilated patients allows to evaluate the lung condition and to monitor the respiratory function during the clinical course of the patient in the intensive care unit. It is a fundamental complement to the clinical evaluation in patients with respiratory failure aiding diagnosis of aetiology (e.g. restrictive vs. obstructive disease) and in all patients requiring recognition and prompt treatment of critical events such as bronchospasm or endotracheal tube displacement. Moreover, increasing attention has been dedicated to limit the deleterious effects of mechanical ventilation (i.e. ventilator-induced lung injury) $(3,4)$. In this context, monitoring airway pressure is essential to characterize, diagnose and assess the therapeutic response.

The two main elements of respiratory mechanics are resistance and compliance. The purpose of this article is to measure these at the bedside and to explain the rationale. We will only focus on passive mechanically ventilated patient with no respiratory effort.

Submitted: September 7th, 2019

Accepted: October 10th, 2019

Published online: November 22, 2019

Corresponding author

Marco Giani

Dipartimento di Emergenza-Urgenza

Rianimazione Generale, Ospedale San Gerardo, ASST Monza

Via Pergolesi 33, 20900

Monza, Italy

marco.giani@unimib.it

\section{Theory and definition}

The respiratory system can be assimilated to a closed system, comprising a flow generator (the mechanical ventilator), a conductive system (endotracheal tube and lower airways) and an elastic system (the alveoli). Several variables are measured by the mechanical ventilator, and many more can be derived:

- The pressure at airway opening, which is normally measured at the proximal tip of the endotracheal tube or in the ventilator itself. More often this pressure is referred to as "airway pressure" $\left(P_{\text {aw }}\right)$ : albeit widely used (and we will also use this nomenclature), it is worth noting that, whenever a flow is present a pressure gradient must exist. Therefore, airway pressure will not be uniform along the airway, by definition.

- The airflow or flow: this is the flow (amount of volume in the time unit) leaving the ventilator towards the airway opening and the alveoli (positive flow, inspiration) or vice versa (negative flow, expiration). It is usually expressed as $\mathrm{L} / \mathrm{min}$ or $\mathrm{L} / \mathrm{s}$.

- The volume: this is calculated from the ventilator as the digital integration of flow over time and it is assumed to be expanding the alveoli. This might not always be the case, for example, in the presence of leaks. It is normally expressed in $\mathrm{mL}$.

The measurement of pressure, flow and volume allows to calculate the two fundamental parameters describing the respiratory system mechanics:

- $\quad$ Resistance of the respiratory system $\left(R_{\mathrm{rs}}\right)$ describes the opposition to a gas flowing into the airways during inspiration. Resistance comprises two elements: the "ohmic" resistance (which depends on the frictional force opposing 
airflow) and the viscoelastic resistance (due to the resistance to deformation of the respiratory system) (5). We will consider both components as a single entity. $R_{\mathrm{rs}}$ is calculated as the ratio between the pressure difference $(\Delta P)$ at the two ends of the airways (the ventilator unit and the alveoli, in this case) and the resulting flow rate. $R_{\mathrm{rs}}=\Delta P /$ Flow. $R_{\mathrm{rs}}$ is usually expressed as $\mathrm{cmH}_{2} \mathrm{O} / \mathrm{L} / \mathrm{s}$.

- The compliance of the respiratory system $\left(\mathrm{Cpl}_{\mathrm{rs}}\right)$ describes the elastic property of the respiratory system, including the lung and the chest wall. $\mathrm{Cpl}_{\mathrm{rs}}$ is the ratio between the inspired volume and the corresponding change in pressure. $\mathrm{Cpl}_{\mathrm{rs}}$ is usually expressed as $\mathrm{mL} / \mathrm{cmH}_{2} \mathrm{O}$. The elastic properties of the respiratory system can also be described as elastance $(E)$, which is the reciprocal of compliance and expressed as $\mathrm{CmH}_{2} \mathrm{O} / \mathrm{mL}$.

All the variables expressed above are linked by a very simple equation, known as the Equation of Motion of the respiratory system, according to which pressure at the airway opening ( $P_{\text {aw, }}$ displayed on the ventilator screen) equals, at any instant of the breathing cycle, the sum of the elastic $\left(P_{\text {el }}\right)$ and resistive $\left(P_{\text {res }}\right)$ pressures, plus the total positive end-expiratory pressure (PEEP, see below), which, being constant throughout the cycle, can be considered as the constant term.

$$
P_{\text {aw }}=P_{\text {res }}+P_{\text {el }}+\text { PEEP }
$$

As stated, $P_{\text {res }}$ is the pressure necessary to overcome the resistance to airflow, whereas $P_{\mathrm{el}}$ is the pressure due to the elastic recoil of the respiratory system when a certain volume $(V)$ is inflated.

$$
\begin{aligned}
& P_{\text {res }}=\text { Flow } * R_{\text {rs }} \\
& P_{\text {el }}=V / C_{\text {rs }}
\end{aligned}
$$

Figure 1 graphically describes $P_{\text {aw }}$ and its components during the respiratory cycle.

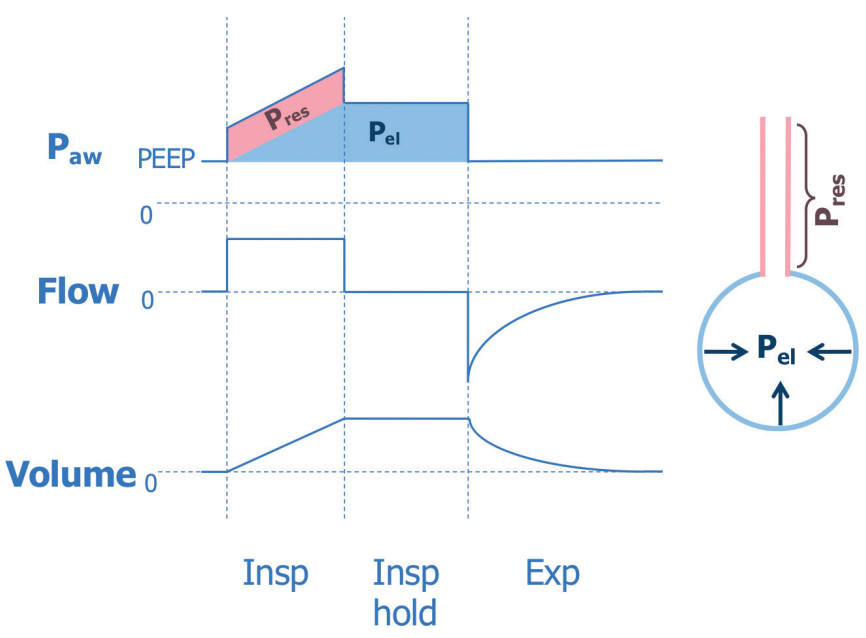

Fig. 1 - Airway pressure $\left(P_{\text {aw }}\right)$ and flow during a volume-controlled breath. PEEP, positive end-expiratory pressure; $P_{\text {res' }}$, resistive pressure; $P_{\mathrm{el}}$, elastic pressure.

Describing respiratory mechanics in a spontaneously breathing patient is beyond the scope of the current article.
However, in the presence of patient's effort, the equation is simply modified by adding the term $P_{\text {musc }}$ (i.e. the pressure generated by the patient's respiratory muscles) in the equation:

$$
P_{\text {ao }}=P_{\text {res }}+P_{\text {el }}+P_{\text {musc }}+\text { PEEP }
$$

It might actually be more correct to consider Eq. [1] as a simplification of the more general Eq. [4] with the term $P_{\text {musc }}=0$.

\section{Measuring respiratory mechanics during volume-controlled ventilation}

Ventilators in volume-controlled modality deliver a constant flow rate during inspiration (i.e. square flow waveform) until the set volume (tidal volume, $V_{\mathrm{T}}$ ) is entirely delivered. Therefore, $P_{\text {aw }}$ progressively increases, starting from the set PEEP level. This is due to the presence of $P_{\text {res }}$ (normally constant during inspiration, given the use of a constant flow) and $P_{\text {el, }}$ which increase gradually due to the increase of the lung volume $\left(P_{\mathrm{el}}=\mathrm{V} / \mathrm{Cpl} \mathrm{rs}_{\mathrm{rs}}\right)$. At the end of tidal volume insufflation, peak inspiratory pressure $\left(P_{\text {peak }}\right)$ is reached. $P_{\text {peak }}$ represents the highest pressure during the ventilatory cycle, and it is the sum of pressures resulting from inspiratory flow resistance (resistive pressure, $P_{\text {res }}$ ), elastic recoil of the lung and chest wall (elastic pressure, $P_{\mathrm{el}}$ ) and the alveolar pressure present at the beginning of the breath (PEEP).

$$
P_{\text {peak }}=\text { Flow }^{*} R_{\mathrm{rs}}+\mathrm{V} / \mathrm{Cpl} \mathrm{rs}_{\mathrm{rs}}+\text { PEEP }
$$

After interruption of the inspiratory flow, when the expiratory valve is still closed, the plateau pressure value is reached $\left(P_{\text {plat }}\right)$. When gas flow is zero, there is no resistive pressure between the alveoli and the ventilator; therefore, plateau pressure equals alveolar pressure.

$$
P_{\text {plat }}=V / \mathrm{Cp}_{\mathrm{rs}}+\mathrm{PEEP}
$$

Hence, the difference between peak inspiratory pressure $\left(P_{\text {peak }}\right)$ and plateau pressure $\left(P_{\text {plat }}\right.$ allows the calculation of the resistance of the respiratory system:

$$
\begin{aligned}
& P_{\text {peak }}-P_{\text {plat }}=\left(\text { Flow }^{*} R_{\mathrm{rs}}+V / \mathrm{Cpl}_{\mathrm{rs}}+\mathrm{PEEP}\right)-\left(\mathrm{V} / \mathrm{Cp} \mathrm{rs}_{\mathrm{rs}}\right. \\
& +\mathrm{PEEP})=\text { Flow }^{*} R_{\mathrm{rs}} \\
& R_{\mathrm{rs}}=\left(P_{\text {peak }}-P_{\text {plat }}\right) / \text { Flow }
\end{aligned}
$$

Figure 2 shows the pressure drop during an inspiratory hold and an example of how to calculate $R_{\mathrm{rs}}$ during volumecontrolled ventilation.

Since $P_{\text {plat }}$ and PEEP reflect alveolar pressure at the end of inspiration and expiration, respectively, their difference (also known as "driving pressure", DP) allows the calculation of respiratory system compliance:

$$
\begin{aligned}
& P_{\text {plat }}-\text { PEEP }=\left(V / C p I_{r s}+\text { PEEP }\right)-\text { PEEP }=V / C p I_{r s} \\
& C p I_{r s}=V /\left(P_{\text {plat }}-\text { PEEP }\right)=V / D P
\end{aligned}
$$

During expiration, alveolar pressure decreases from $P_{\text {plat }}$ towards PEEP. The ventilator is designed to adjust the opening of the expiratory valve to maintain the set PEEP during the expiratory phase. However, if an expiratory flow is still present at the end of the expiration, the alveolar pressure 


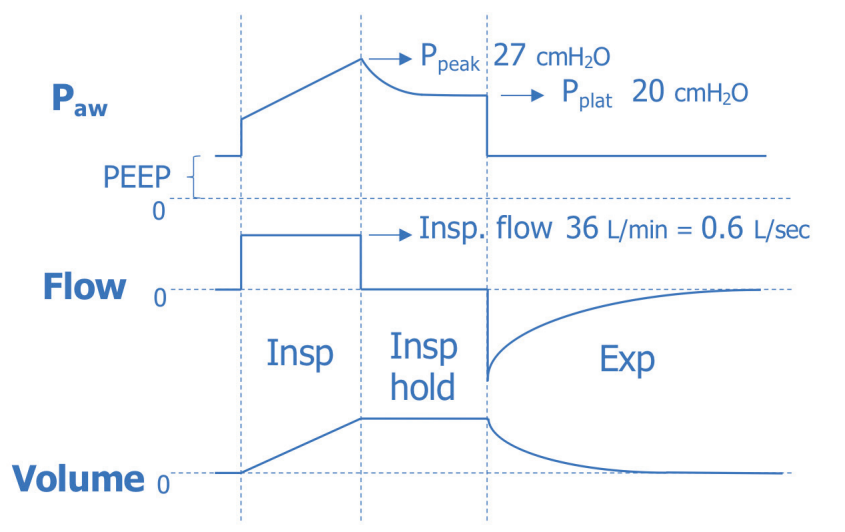

$$
\mathrm{R}_{\mathrm{rs}}=(27-20) / 0.6=11.67 \mathrm{cmH}_{2} \mathrm{O} / \mathrm{L} / \mathrm{sec}
$$

Fig. 2 - An example of how to measure resistance of the respiratory system $\left(R_{\mathrm{rs}}\right)$ during volume-controlled ventilation. During an inspiratory hold, the drop in airway pressure $\left(P_{\text {aw }}\right)$ corresponds to the amount of pressure generated during inspiration to overcome the resistance of the respiratory system. Insp, inspiratory phase; Exp, expiratory phase; PEEP, positive end-expiratory pressure; $P_{\text {res }}$, resistive pressure; $P_{\text {el' }}$ elastic pressure; $P_{\text {peak }}$, peak inspiratory pressure; $P_{\text {plat' }}$, plateau pressure.

will exceed the pressure at the airway opening. For this reason, to measure the real alveolar pressure at the end of the expiration, an expiratory hold is required to measure the total PEEP $\left(\right.$ PEEP $\left._{\text {tot }}\right)$. PEEP tot $_{\text {is }}$ the sum of the PEEP set at the ventilator by the clinician and the intrinsic PEEP (PEEP), which results from incomplete emptying of the alveoli at the end of the expiratory time $(6,7)$. All the calculations indicat-

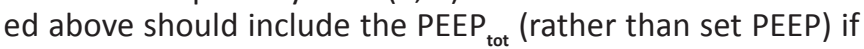
these differ.

Figure 3 reports an example of inspiratory and expiratory holds during volume-controlled ventilation to measure $\mathrm{Cpl}_{\mathrm{rs}}$.

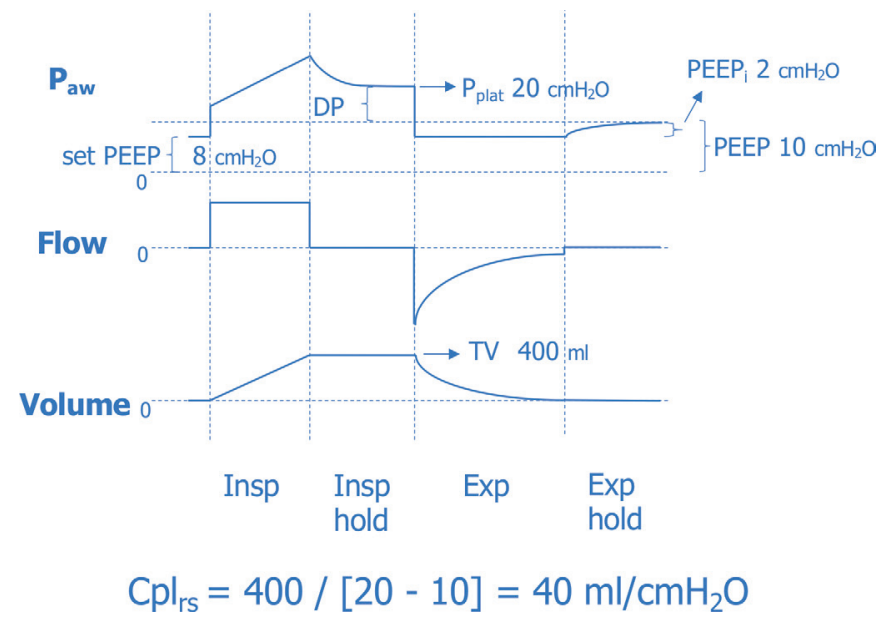

Fig. 3 - An example of how to measure compliance of the respiratory system $\left(\mathrm{Cpl}_{\mathrm{rs}}\right)$ during volume-controlled ventilation (VCV). Insp, inspiratory phase; Exp, expiratory phase; $P_{\text {aw' }}$ airway pressure; PEEP, positive end-expiratory pressure; $P_{\text {plat' }}$, plateau pressure.

\section{Clinical and practical aspects}

Measuring plateau pressures requires an inspiratory hold (flow $=0$ ), which can be part of each tidal breath if an inspiratory pause time is set. However, for a more accurate measurement, a longer (e.g. 2-3 seconds) inspiratory hold (manually performed) allows better equilibration of pressure at the airway opening and at the alveoli.

$R_{\mathrm{rs}}$ is primarily made up of the resistance of the airways and the endotracheal tube, because in normal condition the resistance of the lung tissue is low. $R_{\mathrm{rs}}$ (usually expressed as $\mathrm{CmH}_{2} \mathrm{O} / \mathrm{L} / \mathrm{s}$ ) can only be measured in volume control mode, which provides a constant flow rate during inspiration. It should be considered that $R_{\mathrm{rs}}$ may be influenced by inspiratory flow variations. Therefore, in clinical practice, in order to obtain comparable values over time, a $10 \mathrm{~mL} / \mathrm{kg} / \mathrm{s}$ inspiratory flow should be set at the ventilator (i.e. $70 \mathrm{~kg}=700 \mathrm{~mL} / \mathrm{s}$ $=0.7 \mathrm{~L} / \mathrm{s}=42 \mathrm{~L} / \mathrm{min}$ ). In mechanically ventilated patients with a normal lung and an artificial airway, inspiratory resistances rarely exceed $15 \mathrm{cmH}_{2} \mathrm{O} / \mathrm{L} / \mathrm{s}$ (8). A narrow endotracheal tube or a heat and moisture exchanger may cause an increase in resistances, which increases with flow in an exponential relationship. Incorrect positioning or kinking of the endotracheal tube may also increase resistances. Increased airway resistance also occurs in some pathological conditions such as asthma or chronic obstructive pulmonary disease.

As recommended for the measurement of $\mathrm{Cpl}_{\mathrm{rs}}$, when measuring resistances, the manual holds must be long enough to allow equilibration of pressure at the airway opening with the alveolar one. For this reason, plateau pressures automatically displayed by the ventilators can be inaccurate, especially when resistances of the respiratory system are high.

In order to obtain reliable measurements of respiratory mechanics during controlled mechanical ventilation, the patient must be perfectly adapted (i.e. passive). An unstable pressure tracing during the inspiratory hold suggests the presence of patient effort, which may induce misinterpretation of respiratory mechanics. In these situations, a brief increase of patient sedation may allow a more accurate assessment.

In healthy mechanically ventilated patients $\mathrm{Cpl}$ is around $50-60 \mathrm{~mL} / \mathrm{cmH}_{2} \mathrm{O}$, or $0.7-1 \mathrm{~mL} / \mathrm{cmH}_{2} \mathrm{O} / \mathrm{kg}$ (8). During general anaesthesia in healthy subjects, a reduction in $\mathrm{Cpl}_{\mathrm{rs}}$ mainly depends on decrease of aerated lung volume (9). Decreased compliance is common in pathological conditions such as acute respiratory distress syndrome (ARDS), atelectasis, pneumothorax, lung fibrosis or chest wall stiffness.

ARDS patients typically have a reduced $\mathrm{Cpl}$ that decreases with ARDS severity; therefore, monitoring compliance in ARDS patients can provide information about the volume of the aerated lung (10). It must be noted that in many conditions, including ARDS, patients' airway closure may strongly influence the assessment of static respiratory mechanics (11).

Contrarily, an increase in compliance occurs in the case of lung emphysema.

In contrast to what we explained for the measurement of the resistances, $\mathrm{Cpl}_{\mathrm{rs}}$ can also be measured during pressurecontrolled ventilation. It must be noted that inspiratory pressure during PCV, despite having a flat shape, does not always reflect plateau pressure (12). Figure 4 shows an 


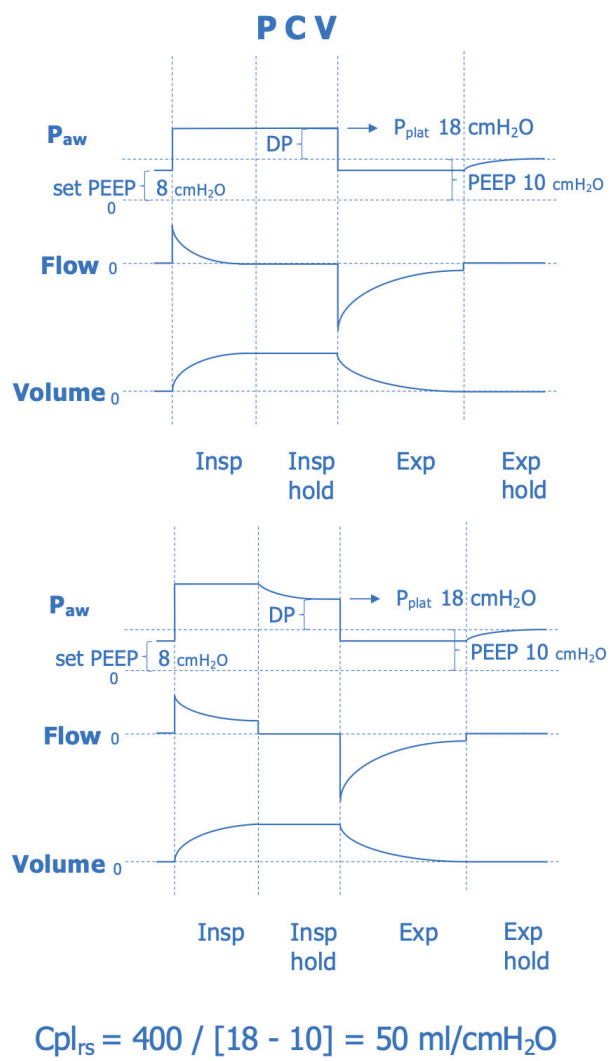

Fig. 4 - Measurement of respiratory system compliance $\left(\mathrm{Cpl}{ }_{\mathrm{rs}}\right)$ during pressure-controlled ventilation (PCV). Manual holds are displayed at the end of inspiration (Insp) and expiration (Exp). The first example (upper panel) shows a case where the inspiratory flow reaches zero before the inspiratory hold, thus no pressure drop is observed. In the second patient (lower panel), the inspiratory hold interrupts a residual inspiratory flow, thus airway pressure decreases due to the zeroing of the resistive pressure component. $P_{\text {aw' }}$ airway pressure; $P_{\text {plat' }}$ plateau pressure; PEEP, total peep; DP, driving pressure.

example of measurement of compliance during pressurecontrolled ventilation.

However, when assessing respiratory mechanics, we recommend to set the ventilator in volume-controlled ventilation in order to visualize all pressures at a glance and to measure both $R_{\mathrm{rs}}$ and $\mathrm{Cpl}_{\mathrm{rs}}$ with just one inspiratory and one expiratory hold.

Figure 5 provides some examples of how to interpret a modification of airway pressures during volume-controlled ventilation. An increase of resistance is evident when $P_{\text {peak }}$ increases without modification of $P_{\text {plat }}$ (Patient A). A reduction of compliance is suggested in the case of similar increase of $P_{\text {peak }}$ and $P_{\text {plat }}$ (Patient B). The last example shows a patient with both increased $R_{\mathrm{rs}}$ and decreased $\mathrm{Cpl}_{\mathrm{rs}}$ : the peak to plateau pressure drop is high but $P_{\text {plat }}$ is also increased (Patient C). This visual approach to pressure curve can be particularly useful in case of critical events.

\section{Conclusion}

Measuring respiratory mechanics is essential in critical care patients in order to avoid complication and to face any

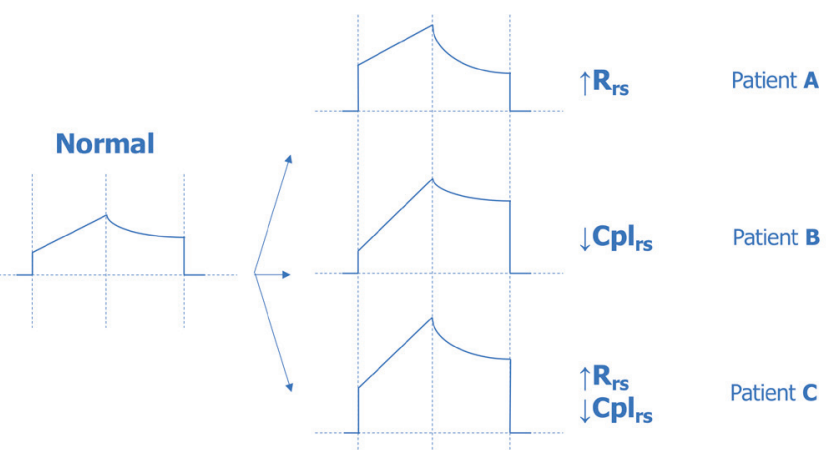

Fig. 5 - Modification of the pressure curve at the ventilator in case of increase of resistance $\left(R_{r s}\right.$ Patient $\left.\mathrm{A}\right)$, decrease of compliance $\left(\mathrm{Cpl}_{\mathrm{rs}}\right.$, Patient B) or both (Patient C).

ventilation problem. Intensivists should perform inspiratory and expiratory holds at the ventilator during every clinical assessment of a ventilated patient. Measuring respiratory mechanics should be considered as important as assessing fluid balance, deciding antibiotic therapy or choosing sedation.

\section{Disclosures}

Conflict of interest: The authors have no conflicts of interest to declare.

Financial support: the author declare no financial support

\section{References}

1. Hess DR, Medoff BD, Fessler MB. Pulmonary mechanics and graphics during positive pressure ventilation. Int Anesthesiol Clin. 1999;37(3):15-34.

2. Lucangelo $U$, Bernabé $F$, Blanch L. Lung mechanics at the bedside: make it simple. Curr Opin Crit Care. 2007;13(1):64-72.

3. Slutsky AS, Ranieri VM. Ventilator-induced lung injury. N Engl J Med. 2013;369:2126-2136.

4. Uhlig S, Ranieri M, Slutsky AS. Biotrauma hypothesis of ventilator-induced lung injury. Am J Respir Crit Care Med. 2004; 169:314-315.

5. Rubini A. Flow and volume dependence of resistive pressures dissipation in the respiratory system. Minerva Pneum. 2017; 56(4):249-253.

6. Marini JJ. Dynamic hyperinflation and auto-positive endexpiratory pressure: lessons learned over 30 years. Am J Respir Crit Care Med. 2011;184(7):756-762.

7. Blanch L, Bernabé F, Lucangelo U. Measurement of air trapping, intrinsic positive end-expiratory pressure, and dynamic hyperinflation in mechanically ventilated patients. Respir Care. 2005;50(1):110-123.

8. Maclntyre NR. Evidence-based guidelines for weaning and discontinuing ventilatory support. Chest. 2001;120:375S-396S.

9. Grieco DL, Russo A, Antonelli M. Lung volumes, respiratory mechanics and dynamic strain during general anaesthesia. $\mathrm{Br} \mathrm{J}$ Anaesth. 2018 Nov 1;121(5):1156-1165.

10. Gattinoni L, Marini JJ, Brochard L. The "baby lung" became an adult. Intensive Care Med. 2016;42:663-673.

11. Chen L, Del Sorbo L, Brochard L. Airway closure in acute respiratory distress syndrome: an underestimated and misinterpreted phenomenon. Am J Respir Crit Care Med. 2017 May 30;197(1):132-136.

12. Sosio S, Bellani G. Plateau Pressure during Pressure Control Ventilation. Abtpn [Internet]. 2019 Oct 22;6(1):76-77. 